RESEARCH ETHICS

\title{
Equitable treatment for HIV/AIDS clinical trial participants: a focus group study of patients, clinician researchers, and administrators in western Kenya
}

\author{
D N Shaffer, V N Yebei, J B Ballidawa, J E Sidle, J Y Greene, E M Meslin, S J N Kimaiyo, \\ W M Tierney
}

J Med Ethics 2006;32:55-60. doi: 10.1136/jme.2004.011106

See end of article for authors' affiliations

Correspondence to: Dr D N Shaffer, The Walter Reed Project, USAMRU-K, Unit 64109 APO AE 09831-4109, Kenya; dshaffer@kericho. mimcom.net

Received 1 November 2004 In revised form 29 March 2005

Accepted for publication 6 April 2005
Objectives: To describe the concerns and priorities of key stakeholders in a developing country regarding ethical obligations held by researchers and perceptions of equity or "what is fair" for study participants in an HIV/AIDS clinical drug trial.

Design: Qualitative study with focus groups.

Setting: Teaching and referral hospital and rural health centre in western Kenya.

Participants: Potential HIV/AIDS clinical trial participants, clinician researchers, and administrators.

Results: Eighty nine individuals participated in a total of 11 focus groups over a four month period. The desire for continued drug therapy, most often life long, following an HIV/AIDS clinical trial was the most common priority expressed in all focus groups. Patients with and without HIV/AIDS also thought subsidisation of drug therapies and education were critical forms of compensation for clinical trial participation. Financial incentives were considered important primarily for purchasing drug therapy as well as obtaining food. Patients noted a concern for the potential mismanagement of any money offered. Clinician researchers and administrators felt strongly that researchers have a moral obligation to participants following a trial to provide continued drug therapy, adverse event monitoring, and primary care. Finally, clinician researchers and administrators stressed the need for thorough informed consent to avoid coercion of study participants.

Conclusions: Kenyan patients, clinician researchers, and administrators believe that it would be unfair to stop antiretroviral therapy following an HIV/AIDS clinical trial and that researchers have a long term obligation to participants.

\begin{abstract}
"I have been used like a guinea pig, so how does he just leave me without compensation?" (Moi Hospital General Medicine Clinic male)
\end{abstract}

$\mathrm{R}$ ecent experiences with HIV/AIDS clinical trials within African and other developing countries have prompted economical issues that arise when human subjects participate in research. ${ }^{1-13}$ An especially challenging issue is that of ensuring vulnerable individuals are treated fairly both during and after a trial. Although considerable discussion has occurred regarding ethical issues arising during a study, the particular circumstances surrounding research sponsored and conducted by investigators from economically developed countries and performed in economically underdeveloped countries have focused increased attention on post-study issues. ${ }^{1-4} 8-111^{13}$ This latter concern is sometimes described as an issue of post-trial obligations: what obligations do trial sponsors and researchers have, if any, to participants at the end of a study? ${ }^{14} 15$

Recommendations from national bioethics commissions examining the ethics of research in economically developed countries have come to similar conclusions: that researchers and sponsors do have post-trial obligations to participants. $^{3410}$ For example, the United States' National Bioethics Advisory Commission (NBAC) recommended that "researchers and sponsors in clinical trials should make reasonable, good faith efforts before the initiation of a trial, to secure, at its conclusion, continued access for all participants to needed experimental interventions that have been proven effective for the participants."
Recently, international attention has focused on aspects of international research including important roles played by communities in designing and assessing clinical trials. ${ }^{16} 17$ However, although there are nearly 100 domestic and international guidelines for the protection of human subjects, the vast majority are silent on issues regarding community roles in clinical research..$^{410}$ Of guidelines addressing concepts of justice, equity, and ethical obligations that arise at the end of trials, the more influential are western in their origin and are therefore based upon beliefs and principles of the developed world. ${ }^{45} 810$ 18-20 Moreover, even though research guidelines are now being written by developing countries, few data, if any, exist describing what individuals (that is, study participants, clinician researchers, and institutional administrators) from developing countries think about the issue of post-trial obligations..$^{21-23}$

We conducted 11 focus group sessions consisting of potential HIV/AIDS clinical trial participants, clinician researchers, and administrators at a teaching and referral hospital and rural health centre in western Kenya. In light of a developing international HIV/AIDS research programme, we aimed to describe the concerns and priorities of these key stakeholders regarding ethical obligations held by researchers and perceptions of equity or "what is fair" or "fairness" for study participants both during and after an HIV/AIDS clinical drug trial. We explored the ethical issues and gathered primary data for use in joint decision making by key stakeholders from both developing and industrialised countries as they consider clinical trial protocols and develop research guidelines. 


\section{PARTICIPANTS AND METHODS}

\section{Study sites and participant recruitment}

The study was conducted at the Moi Teaching and Referral Hospital ("Moi Hospital") in Eldoret, Kenya, and Mosoriot Rural Health Center ("Mosoriot Center"). Located in the northern Rift Valley, Moi Hospital is the primary referral hospital for western Kenya serving a catchment area of approximately 13 million people and is affiliated with Moi University Faculty of Health Sciences. The Mosoriot Center serves a widespread rural population of approximately 40000 people and is located approximately $30 \mathrm{~km}$ southeast of Eldoret. After approval was obtained from the Moi University Faculty of Health Sciences Institutional Research and Ethics Committee and the Indiana University Institutional Review Board, participants were recruited for three focus group categories: potential clinical trial participants, clinician researchers, and administrators.

At Moi Hospital, we conducted two focus groups (one male and one female) among patients receiving care in the adult HIV/AIDS clinic, representing potential HIV/AIDS clinical trial participants, and two analogous focus groups among patients without documented HIV infection receiving care in the adult General Medicine clinic. In order to explore a more rural population, we conducted four similar focus groups in both HIV/AIDS and General Medicine clinics at Mosoriot Center. Separate male and female focus groups were held to facilitate open and free discussion in recognition of the stigma associated with HIV/AIDS and cultural gender issues that may influence discussion in combined focus groups.

We recruited patients from a sample of approximately 800 patients in the HIV/AIDS clinic (mean age 36 years, 65\% female) and 2500 in the General Medicine clinic (demographics unavailable) at Moi Hospital and 250 patients in the HIV/AIDS clinic (mean age 36 years, 73\% female) and 15000 in the General Medicine clinic (mean age 31 years, 68\% female) at Mosoriot Center. Clinic nurses recruited patients presenting to the HIV/AIDS and General Medicine clinics. Patients were informed of the study objectives, and recruitment proceeded until 15 people volunteered for each focus group. Balancing of patient focus groups was based upon HIV history (present or absent by patient report), clinic site (rural versus urban), and sex (male versus female). None of the patients had participated in therapeutic HIV/AIDS clinical trials.

Two clinician researcher focus groups and one administrator focus group were held at Moi Hospital. Study investigators recruited clinician researchers from the approximately 120 faculty members of the Moi University Faculty of Health Sciences. The following administrators were invited: the Deputy Vice Chancellors of Moi University, the Dean of the Moi University Faculty of Health Sciences Medical School, the Dean of the Moi University School of Public Health, the Director of the Moi Teaching and Referral Hospital, and representatives from the Institutional Research and Ethics Committee secretariat.

We excluded individuals less than 18 years old, those not providing consent or not interested in participating, and those unable to communicate effectively in Kiswahili or English. A written Kiswahili and/or English informed consent document was provided to all focus group participants and a verbal, witnessed informed consent process was used. Invited participants presenting for the focus group sessions were given 200 Kenyan Shillings (approximately US\$2.40) to cover transportation expenses and were provided with a meal. Clinician researchers and administrators willing to participate provided informed consent and were offered a meal following the focus group.

\section{Study instrument and focus group leaders}

We developed scripts to guide focus group discussions regarding the ongoing ethical debate surrounding equitable treatment for clinical trial participants in developing countries. Two separate guides were developed: one for patients and one for clinician researchers and administrators. Four experienced focus group facilitators, fluent in both Kiswahili and English, and a clinical researcher were trained by a medical anthropologist and conducted the 11 focus groups. The script for potential clinical trial participants was piloted on two additional patient focus groups, and the results were critiqued by the medical anthropologist, clinical researcher, and the four Kenyan facilitators.

Facilitators used a script containing neutral language to broadly ask focus group participants to discuss issues concerning treatment of patients during and following an HIV/AIDS drug trial. Discussions centred on two standard introductions (one for patient and one for clinician research and administrator focus groups) associated with a hypothetical HIV/AIDS drug trial (box 1). Following introduction and elaboration of a hypothetical trial, three primary questions were asked:

1. Is it fair for the doctor to treat patients for only as long as the study lasts?

2. What other things should the doctor think about as he decides how long to treat patients from whom he is learning after the actual study is completed?

3. What do you want to be absolutely certain that those who study this conversation understand? What do you want to be sure they hear?

The script for the clinician researchers and administrators was similar to the script for patients but focused on the perspective of the researchers and administrators designing

\section{Box 1 Focus group introductions and HIV/AIDS} research

1. HIV/AIDS and General Medicine focus groups "The doctors here are trying to understand how different medicines work to help people who are sick with HIV/AIDS. Some of these medicines may save people's lives, but to work best they need to be taken for long periods of time. Many of these medicines are very expensive, but are helpful only as long as they are being taken regularly. Some of our researchers feel that patients in this type of study should not lose access to the drugs once the study is over; others feel that the cost of drugs means that we cannot keep patients on them once the study has been completed. Research, of course, is necessary if the doctors are to find new and better drugs. It is very important to understand what YOU AS PATIENTS think and feel about this issue."

2. Clinician researcher and administrator focus groups

"Suppose physician researchers here are trying to understand through clinical trials how a particular medicine works to help HIV/AIDS patients. You are aware of the limited budgets available for such research. In order to learn about the drug safety and effectiveness, the doctor will, of course, have to observe the subjects who are being treated for some specified length of time."

Note

Focus group facilitators used standardised introductions spoken in Kiswahili and/or English depending upon the needs of the focus group participants. Further elaboration using a hypothetical HIV/AIDS drug trial was made as necessary. 
and reviewing such studies. In addition, the script for the patient focus groups included a preliminary scenario surrounding "fairness" and bargaining in a market setting in an attempt to have the participants understand and consider the concept of equity. When leading discussions, facilitators used a non-directive approach with prompts (for example, "fairness" to study participants as well as other benefit to Kenyans, potential participant compensations (patient focus groups), and rationale for study versus post-trial obligations (clinician researcher and administrator)), where necessary.

\section{Coding and analyses}

Coding and analyses of data followed a stepwise procedure:

- Sessions were audio taped and transcribed verbatim by a secretary fluent in both Kiswahili and English but blinded to the goals of the study.

- Transcripts were independently reviewed by a medical anthropologist, a Kenyan general internist, an American general internist with extensive experience in Kenya, and a Kenyan psychologist.

- Each rater used a two step process in reviewing the transcripts. Firstly, transcripts were read and developing themes were identified. These themes were then aggregated into lists. Phrases and quotations were highlighted as illustrations of emerging themes.

- Finally, all rater themes and lists were independently reviewed and aggregated according to the transcript questions.

As our goal was to understand the perspective of potential clinical trial participants and individuals likely to be involved in conducting or reviewing clinical trials, we aggregated factors as apparently understood by these groups.

\section{RESULTS}

\section{Focus groups composition}

During four months (February to May, 2003), 11 focus groups were conducted at Moi Hospital and Mosoriot Center (table 1). Thirty eight patients (63\% of those invited) from four HIV/AIDS clinics and 35 patients (58\%) from four General Medicine clinics participated. Eleven clinician researcher (44\%) and five administrators (71\%) participated.

\section{Lifelong antiretroviral therapy}

All focus groups unanimously felt it would be unfair for antiretroviral therapy to be withdrawn following a clinical

\begin{tabular}{|c|c|c|}
\hline Focus group and gender & Site & $\begin{array}{l}\text { No of participants/ } \\
\text { no invited }\end{array}$ \\
\hline \multicolumn{3}{|l|}{ HIV/AIDS patients } \\
\hline Female & Moi Hospital & $10 / 15$ \\
\hline Male & Moi Hospital & $7 / 15$ \\
\hline Female & Mosoriot & $13 / 15$ \\
\hline Male & Mosoriot & $8 / 15$ \\
\hline \multicolumn{3}{|c|}{ General medicine patients } \\
\hline Female & Moi Hospital & $11 / 15$ \\
\hline Male & Moi Hospital & $7 / 15$ \\
\hline Female & Mosoriot & $9 / 15$ \\
\hline Male & Mosoriot & $8 / 15$ \\
\hline \multicolumn{3}{|l|}{ Clinician researchers } \\
\hline Combined ( $60 \%$ male) & Moi Hospital & $5 / 13$ \\
\hline Combined ( $83 \%$ male) & Moi Hospital & $6 / 12$ \\
\hline \multicolumn{3}{|c|}{ Administrators } \\
\hline Combined ( $100 \%$ male) & Moi Hospital & $5 / 7$ \\
\hline
\end{tabular}

trial (table 2). A typical response was expressed by one individual with HIV/AIDS: "I feel that since there is nothing that is impossible, maybe that doctor may have those drugs and he wants to use them on me as a research and they may work properly. If he takes me to the laboratory and I may find there is no virus, there he has a right to discontinue the use once he is sure that I am healed. But if I am not completely healed, he should not stop the drugs" (Mosoriot Center HIV/ AIDS clinic male). This consensus was based upon a fear of illness and death associated with a lack of funds to continue therapy. One clinician researcher reported on behalf of trial participants: "Because I will get into this trial, I get better, and then afterwards I am going to die. You have promised me life and then you take it back; that's not fair" (clinician researcher, group 1).

Patients generally felt that antiretroviral therapy should be continued for duration of life. This position centred on a desire for wellness. One patient insisted that therapy should continue until the patient is healed, "lifelong", or " til eternity": "If there is no cure, then where are we? The drugs we are researching on are to sustain the patient; they just give you strength to prolong your life. So, you are supposed to get these drugs until time immemorial" (Moi Hospital General Medicine clinic male). Patients with HIV/AIDS questioned how antiretroviral therapy could be discontinued following a study, particularly if the patient was ill: "If a patient is still sick, how will you stop the drugs?" (Moi Hospital General Medicine clinic female). Finally, administrators felt that stopping potentially lifesaving therapy at the end of a trial could result in loss of trust in the doctor-patient relationship and in unwillingness by Kenyans to participate in future trials.

Caveats were noted where cessation of antiretroviral therapy would be appropriate. Reflecting a valued patientdoctor relationship, the main scenario where patients felt stopping therapy would be considered fair would be if/when doctors recommended that therapy be stopped. Patients felt therapy could be stopped following a trial if a cure were to become available or if drug resistance developed. Clinician researchers felt that uncertain drug efficacy and serious drug toxicity were situations where it may be justified to discontinue therapy. Clinician researchers also felt that

Table 2 Concerns regarding participation in HIV/AIDS clinical drug trials

\begin{tabular}{ll}
\hline Primary topic & Concerns \\
\hline Antiretroviral continuation & Lifelong duration \\
& Until physician tells patient to stop \\
& Until a cure is available \\
& Unless side effects or resistance mandate \\
& cessation \\
& Duration clearly explained in consent \\
process & For trial participation \\
Monetary compensation & To purchase antiretroviral therapy \\
& To purchase food \\
& Awareness of potential abuse \\
& Patient/community education an \\
& additional compensation \\
& General health care \\
Follow up and treatment & Ilness discovered during the trial \\
& latrogenic adverse events \\
& To patient and community \\
& For patients "helping" investigators \\
Moral obligation & For benefits investigators acquire \\
& For thorough informed consent \\
& Paternalistic need to protect patient \\
& Trial representation of all socioeconomic \\
& strata \\
\hline &
\end{tabular}


therapy could be discontinued at some point following the trial if participants were fully informed before the trial that treatment would be of limited duration and would be stopped once the trial was over.

\section{Monetary and other compensation}

Patients also felt monetary compensation should be given to clinical trial participants (table 2). Monetary compensation was viewed as necessary to provide a direct means for patients to obtain antiretroviral therapy if such therapy was to be discontinued following a study. Additionally, patients felt money could be used to purchase food or other basic necessities of life even if antiretroviral therapy were continued following a trial. In discussing how money could be used to help improve patients' lives, one woman with HIV/ AIDS noted that money might prevent women from engaging in risky activities such as prostitution: "So if they give us money, it will help us and you can't think of going out to prostitute and look for money. But you can eat and you have drugs and you can do anything. You can buy for your children dresses and continue praying that God adds you more days in your life" (Mosoriot Center HIV/AIDS clinic female).

A concern for potential misuse of money was discussed in all patient focus groups. If money was offered, patients felt it should be accompanied by money management education. Patients with HIV/AIDS argued that compensation in other forms (for example, hard goods, foodstuffs, housing, or donations to institutes and supporting organisations) might be preferable to providing currency to individual patients. Additional suggestions for indirect reimbursement included patient and community education to both increase HIV/AIDS awareness and reduce stigma. The significance of money as it relates not only to acquiring medical care but also to survival was reflected by the following: "So people who have money and in fact a lot of money are the ones who are now gaining. They are the ones who are surviving. But those who don't have anything, they are the ones who are dying" (Moi Hospital General Medicine clinic female).

\section{Comprehensive follow up and treatment}

Patients felt that post-trial medical follow up was critical (table 2), reflecting the concern for general medical care as well as care relating to illness discovered during the clinical trial as evidenced by the following: "I think after the doctor has done his research, it's now time for him to deal with the patients. It is wise for him to think about assisting a patient so that his health remains good ..." (Mosoriot Center General Medicine clinic female). Clinician researchers and administrators emphasised the importance of treating iatrogenic adverse effects that occurred during participation in the clinical trial. This concern extended to evaluations following the trial for adverse effects that manifest following study completion. Additionally, clinician researchers emphasised the need for treating subsequent opportunistic infections, even if antiretroviral therapy could not be continued.

\section{Ethical obligation}

A theme stressing the ethical obligation of the researchers to patients participating in clinical trials was evident in all discussions (table 2). Patient focus groups viewed the obligation to patients by researchers (for example, continued care, monetary reimbursement) as appropriate compensation for patients' "helping" the investigation. Clinician researchers stressed the researchers' ethical obligation to participants in return for benefits the study investigator would obtain from the study, as evidenced by the following: "Okay, I am thinking in terms of me as a patient. I have agreed to participate in this study. You people are doing something on me. It's only fair that you compensate me for your research" (clinician researcher, group 1). Administrators more directly noted a post-trial obligation. "It becomes ethically and morally extremely difficult to decide that I was only here for the trial, and I am going to be abandoned at the end of the trial" (administrator). Additionally, "It [the drug] does [work] and you are now going to market [that drug] and get some money out of it. I think there is a moral obligation [to continue treating subjects]. It's not legal, it's not binding, you cannot be taken to court. But it is only fair" (clinician researcher, group 2).

Clinician researchers and administrators felt investigators had a moral obligation not only to the patient (regarding informed consent) but also to the community (regarding recruitment and benefits). These professionals emphasised the necessity for adequate informed consent focusing upon therapy duration, risks/benefits, and alternatives. If a drug will not be continued after the trial "the patient should be aware that this drug is not being administered for life. They will reach a time when it will be stopped. It should be an informed choice" (clinician researcher, group 1). A paternalistic need for "protection" of clinical trial participants was voiced. Based upon discussion of limited participant finances and drug availability, administrators expressed concern that thorough informed consent would be overshadowed by patient desperation for therapy. Finally, clinician researchers and administrators felt that patient selection should be carefully considered, taking into account a potential subject's health status, previous therapy, financial capability to continue therapy once/if it was stopped, and recruitment from all socioeconomic strata of the Kenyan society.

\section{DISCUSSION}

In this focus group study in western Kenya, we found a consistent belief by potential clinical trial participants, clinician researchers, and administrators that it would be unfair to discontinue therapy following an HIV/AIDS clinical trial. If therapy could not be continued, focus group participants universally felt drug therapy should be subsidised or made available through alternative means. Our findings reflect a more broad, growing desire to maximise the welfare of patients in clinical research in developing countries through securing post-trial benefits for them and their communities..$^{2-6} 14$ 18-20 $^{2-}$ The ethical obligation for continued care noted by clinician researchers and administrators was consistent with the desires of potential clinical trial participants and depicted a type of reciprocity: the patient was accepting a risk for knowledge, and in return they expected some form of benefit. ${ }^{14}$ The focus group participants could be expected to describe their preferences to have successful treatment continued because participation in any HIV/AIDS trial might reasonably be regarded as access to a life saving therapy not otherwise available.

It was instructive to find that the focus group responses mirrored the arguments in the bioethics and public policy literature. ${ }^{346-815}$ Focus group participants directly or indirectly expressed concern about the potential for exploitation, whether by unfairly inducing participation, or by making use of human subjects for reasons unrelated to the health needs of the host country. ${ }^{34-9}$ The potential and concern for exploitation we observed is understandable considering the high prevalence of HIV/AIDS in this setting in addition to pervasive poverty and unavailability of antiretroviral drugs.

\section{Themes warranting international consideration}

There were themes observed in our study that are yet unresolved and deserve further consideration. Discussions regarding the advantages and disadvantages of providing financial incentives or rewards for participation in HIV/AIDS 
clinical trials received considerable attention in the focus groups. If not used to purchase therapy, monetary compensation for participation was commonly discussed with regard to purchasing food-a simple yet fundamental need if HIV/ AIDS treatment is to be successful. Additionally, there was a realistic discussion noting that financial incentives that are given be done so along with education or restraints in effort to avoid mismanagement. Finally, patients felt that basic health care should also be provided, especially continued treatment of potentially life threatening opportunistic infections.

Other themes such as education, the doctor-patient relationship, and informed consent warrant discussion. There was a clear desire by patients with and without HIV/ AIDS for education not only for themselves but also for the community in an effort to educate and reduce HIV/AIDS related stigma. Participants viewed clinical trial investigators as a source for such education. This would seem to be a reasonable and manageable benefit from a large scale clinical trial. Also, the high degree of trust in the doctor and his/her influence regarding participation in an HIV/AIDS clinical trial was evident in all patient focus groups, but more so in the rural setting (for example, focus groups concluding it would be "fair" to stop medicines following a trial if the doctor recommended). The uniqueness of the doctor-patient relationship as it relates to this cultural setting and the potential for this relationship to be either enhanced or jeopardised must be more fully explored (that is, perceptions of clinical trial participants receiving "fair" benefits enhancing the relationship and vice versa). This is particularly important because this relationship may differ considerably from the dominant western view where emphasis on separating the traditional healing role of the clinician from the investigative role of the researcher is thought to be an important ethical safeguard. ${ }^{23} 24$

\section{Strengths and limitations}

The greatest strength of our study was in the focus groups selection and study location. We conducted our study in an academic referral hospital in western Kenya and one of its affiliated rural health centres. We involved not only potential HIV/AIDS clinical trial participants, but also clinicians, researchers, and policy makers who will be central to any efforts to conduct clinical trials in this area. To our knowledge, this is the first comprehensive study to evaluate perceptions regarding fair benefits of research in a developing country by the potential participants, clinician researchers, and administrators of the host country.

Limitations in our study warrant recognition. We asked focus group participants a question that, post hoc, may be interpreted as having introduced bias into our reported results. The initial topic the focus group participants considered was "Is it fair for the doctor to treat patients for only as long as the study lasts?" This may be viewed as a nonneutral prompt (a recognised limitation in qualitative research) with an apparent response being "no". However, our study's main interest was whether or not participants would express the opinion that it was fair, as a matter of justice, for investigators to withhold access by patients to HIV/AIDS therapy once a study had been completed. Although the study context was introduced using a question about fairness of post-trial obligations in general, the subsequent discussion centred on the rationale and views associated with the response. If one views this as a nonneutral prompt having introduced bias in our results, then one must also consider the contrary: conducting the study without discussing this critical question would have introduced bias a priori by assuming that no focus group participant would consider the opportunity to receive potentially life saving therapy even if for a limited duration in the context of a clinical trial. One should consider the potential of a non-neutral prompt and magnitude of bias introduced into our results as well as the potential for a priori bias if the question was not discussed.

Finally, the generalisability of our results must be taken in the context of the cultural setting. That is, the views of Kenyans may differ from those in other countries. Also, there is the risk that questions and statements in the facilitators' scripts and focus group transcripts could be misinterpreted when translated between Kiswahili and English. To address this risk, we held two pilot focus groups to be sure that the topics and discussion items were understood in the manner we intended. Additionally, all patient focus group facilitators and the transcriptionist were fluent in both English and Kiswahili. Finally, participation by women in the clinician researcher and administrators' focus groups was disproportionately low, a factor ultimately beyond the control of the study.

\section{CONCLUSIONS}

We conclude that potential clinical trial participants, clinician researchers, and administrators at a referral hospital and rural health centre in western Kenya, typical of developing countries where HIV/AIDS clinical trials may be conducted, felt that it would be unfair to discontinue therapy following an HIV/AIDS clinical trial. Additionally, they thought that researchers have a long term obligation to treat HIV/AIDS clinical trial participants. The rationale behind this beliefwhether fear of death, inability to continue therapy, or an ethical obligation-warrants attention and consideration by those designing HIV/AIDS clinical trials and developing ethical guidelines concerning the conduct of research in developing countries.

\section{ACKNOWLEDGEMENTS}

The authors wish to acknowledge their appreciation to Rose Ayikukwei, Claris Ojwang, Daniel Murgor, and Omar Egesah for their assistance in conducting the patient focus groups at the Moi Teaching and Referral Hospital and Mosoriot Rural Health Center and the patients, clinical researchers, and administrators who were willing to participate in the focus group sessions and share their beliefs.

\section{Authors' affiliations}

D N Shaffer, J E Sidle, J Y Greene, S J N Kimaiyo, W M Tierney, Department of Medicine, Indiana University School of Medicine, Indianapolis, IN, USA

D N Shaffer, J E Sidle, Department of Medicine, Moi University Faculty of Health Sciences, Eldoret, Kenya

V N Yebei, J B Ballidawa, Department of Behavioral Science, Moi University Faculty of Health Sciences, Eldoret, Kenya

E M Meslin, Indiana University Center for Bioethics, Indianapolis, IN, USA

W M Tierney, Regenstrief Institute, Incorporated, Indianapolis, IN, USA

This study was funded by the Department of Medicine of the Indiana University School of Medicine and in part by grant number 1-D43TW01082 from the Fogarty International Center, National Institutes of Health. Other than academic relationship to authors, the Department of Medicine of the Indiana University School of Medicine as the study sponsor had no role in the study design; in the collection, analysis, and interpretation of data; in the writing of the report; or in the decision to submit the paper for publication. The researchers declare independence from the funders of this study.

Competing interests: the authors declare that they have no competing interests, particularly financial, with regard to participation in the focus group study, and/or preparation and submission of this manuscript for review. 


\section{REFERENCES}

1 Pitler LR. Ethics of AIDS clinical trials in developing countries: a review. Food Drug Law J 2002;57:133-53.

2 Killen J, Grady C, Folkers $G$, et al. Ethics of clinical research in the developing world. Nat Rev Immunol 2002;3:210-15.

3 Emanuel EJ, Wendler D, Killen J, et al. What makes clinical research in developing countries ethical? The benchmarks of ethical research. J Infect Dis 2004; 189:930-7.

4 Ethical and policy issues in international research: clinical trials in developing countries. Bethesda, MD: National Bioethics Advisory Commission 2001.

5 Caballero $\mathbf{B}$. Ethical issues for collaborative research in developing countries. Am J Clin Nutr 2002;76:717-20.

6 World Medical Association Declaration of Helsinki: ethical principals for medical research involving human subjects. Edinburgh, UK: World Medical Association, October 2001. Available at http://www.wma.net/e/policy/17 c_3.html (accessed 28 May 2003).

7 Participants in the 2001 Conference on Ethical Aspects of Research in Developing Countries. Ethics. Fair benefits for research in developing countries. Science 2002;298:2133-34.

8 Shapiro H, Meslin E. Ethics issues in the design and conduct of clinical trials in developing countries. N Engl J Med 2001;345:139-42.

9 Brody B. Ethical issues in clinical trials in developing countries. Statist Med 2002;21:2853-58.

10 Nuffield Council on Bioethics. The ethics of research related to healthcare in developing countries. London: Nuffield Council, 2002.

11 Angell $M$. The ethics of clinical research in the third world. N Engl J Med 1997:337:847-9.

12 Lurie $\mathbf{P}$, Wolfe S. Unethical trials of interventions to reduce perinatal transmission of the human immunodeficiency virus in developing countries. N Engl J Med 1997;337:853-6.
13 Varmus $\mathbf{H}$, Satcher D. Ethical complexities of conducting research in developing countries. N Engl J Med 1997;337:1003-5.

14 Benatar SR. The HIV/AIDS pandemic: a sign of instability in a complex global system. J Med Philos 2002;27:163-77.

15 Glantz LH, Annas GJ, Grodin MA, et al. Research in developing countries: taking 'benefit' seriously. Hastings Cent Rep 1998;28:38-42.

16 Moodley K. HIV vaccine trial participation in South Africa-an ethical assessment. J Med Philos 2002;27:197-215.

17 Strauss R, Sengupta S, Quinn S, et al. The role of community advisory boards: involving communities in the informed consent process. Am J Public Health 2001;91:1938-43.

18 UNAIDS. Joint United Nations Programme on HIV/AIDS (UNAIDS). Ethical considerations in HIV preventive vaccine research: UNAIDS Guidance Document. Geneva: UNAIDS, 2000.

19 Macklin R. Justice in international research. In: Kahn J, Mastroianni A Sugarman J, eds. Beyond consent: seeking justice in research. New York, NY: Oxford University Press, 1998:131-65.

20 Guenter D, Esparza J, Macklin R. Ethical considerations in international AIDS vaccine trials: a summary of the consultative process conducted by the Joint United Nations Programme on HIV/AIDS. J Med Ethics 2000;26:37-43.

21 Love S, Okello D. Research bioethics in the Ugandan context II: procedural and substantive reform. Law Med Ethics 2000;28:165-73.

22 Indian Council of Medical Research. Ethical guidelines on biomedical research involving human subjects. New Delhi, 2000.

23 In: Rugemalila JB, Kilama WL, eds. Proceedings of the seminar on health research ethics in Africa. Acta Tropica 2001;78(Suppl 1):S1-126.

24 Kass N, Hyder A. Attitudes and experiences of U.S. and developing country investigators regarding U.S. human subjects regulations. In: Ethical and policy issues in international research, clinical trials in developing countries. National Bioethics Advisory Commission. Vol II. Bethesda, MD: Commissioned Papers, 2001 